\title{
Controlling Omnidirectional-wheel and Correcting by Inertial sensor
}

\author{
Takuma Miyazkai ${ }^{\mathrm{a},}$, and Yuhki Kitazono ${ }^{\mathrm{a}}$ \\ ${ }^{a}$ National Institute of Technology, Kitakyushu College, Japan \\ *Corresponding Author: kitazono@kct.ac.jp
}

\begin{abstract}
In this paper, among the omnidirectional mobile drive systems, we analyzed a moving system of the type using passive rotating rollers. First, we analyze the characteristics of the wheel using the passive rotating roller and obtain the wheel placement condition. Next we give a generalization of the control method of the drive system. Finally, we provide a method to correct drive control using IMU.
\end{abstract}

Keywords: Mechanam Wheel, Drive control, IMU.

\section{Introduction}

In recent years, the spread of robots equipped with moving mechanisms such as Rumba of iRobot and Pepper of SoftBank into general households has been widespread. Demand for domestic robots is expected to increase with the development of AI from now on. As a characteristic of the operating environment of home robots, it is not necessary to consider movement in irregular areas such as outdoor, and since movement on a flat floor like the indoor floor is the main, the robot's It can be said that omnidirectional movement can be realized relatively easily.

Major types of omni-directional movement mechanisms are roughly classified into those of a steering type that changes the direction of travel by mechanically changing the driving direction of the wheels. In this paper, we investigate the omni-directional movement mechanism using the wheel with the passive rotating roller among the omni-directional movement mechanism, which is roughly divided into the drive system with the passively rotating roller shown on the wheel as shown in It was.

In many cases, as a control method of a drive system using a wheel having a passive rotation roller, the rotational speed of the wheel is measured and controlled from an encoder mounted on the wheel. However, in this method, an error occurs when the wheel and the floor slide, and it is impossible to correct this error only by the encoder. Then we calibrate the error caused by the slip of the wheel using an inertial sensor which is not affected by the slip of the wheel and the floor.

In this research, we analyze the characteristics of the drive system using the wheel with the passive rotating roller and the arrangement condition of the wheel, give the generalization of the orthogonal movement and the rotational movement of the drive system, then use these inertial sensors We present a method to correct movement.

\section{Characteristics of wheels with passive rotating rollers}

Wheels with representative passive rotating rollers are the Omni wheel and the Mechanam Wheel. We analyze the action of passive rotating rollers attached to these wheels.

\subsection{Definition the working direction of passive rotating roller}

Due to the passive rotating rollers attached to the wheels there is a direction in which the position of the wheels can move freely even if the wheels are not rotating (Fig. 3). Thus, in order to define the direction in which the passive rotary roller can rotate and move even if the wheel is stationary, the direction of action of the passive rotary roller is defined as the unit direction vector $\vec{e}_{p}$.

$$
\overrightarrow{e_{p}}=\left[\begin{array}{l}
e_{x} \\
e_{y} \\
e_{z}
\end{array}\right] \quad \text { where } \quad\left|\overrightarrow{e_{p}}\right|=1
$$

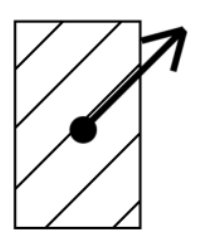

Fig. 1. Passive rotation vector 


\subsection{Characteristics of passive rotating roller}

Due to the action of the passive rotating roller, passive movement occurring when the wheel is stationary can be divided into orthogonal movement and rotational movement. For orthogonal motion, it is possible to move parallel to the unit vector in the direction of action of the passive rotating roller. On the other hand, as for the rotational movement, as shown in Fig. X, when the center of the rotational motion is arranged on a straight line passing through the ground contact point of the wheel and perpendicular to the unit direction vector, the driven wheel performs rotational movement passively You can do. We call this straight line a passive rotation line of the wheel.

\subsection{Placement of multiple wheels}

There is a problem that a robot using a wheel having a passive rotating roller as a moving mechanism moves by a passive rotating roller when the wheel is stationary. For example, when the omni-wheel is arranged as shown in Fig. $\mathrm{X}$, the robot moves passively in the left and right directions in the figure.

Therefore, when any number of wheels having passive rotary rollers are arranged at an arbitrary position on an arbitrary position on the plane, and the positional relationship between the respective wheels is fixed, the arrangement of the wheels for which the drive system does not passively move Find the condition.

First, it is easy to imagine that the condition that the drive system passively orthogonally moves is "the direction vectors of the passive rotating rollers of each wheel are all parallel".

Next, consider the condition under which the drive system passively rotates. As mentioned earlier, if a rotation axis passes through the ground contact point of the wheel and is on a straight line perpendicular to the action vector of the passive roller, the wheel can perform passive rotational movement, so that as shown in FIG., If there is a point where the passive rotation straight lines of all the wheels intersect,

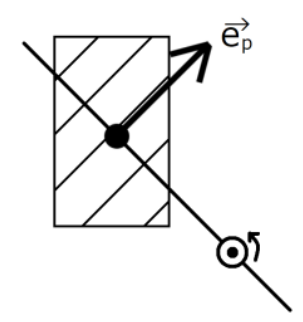

Fig. 2. Passive rotation straight line perpendicular to $\overrightarrow{e_{p}}$

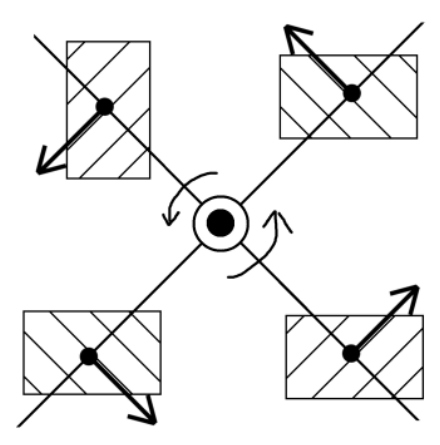

Fig. 3. It is possible to rotate at the intersection of passive rotation straight lines

it is possible to perform a rotational motion about the point. Therefore, it can be said that the condition for the drive system not to passively perform rotational motion is that there is no point where passive rotation lines of all the wheels intersect in one place. (Figure $\mathrm{x}$ )

To summarize the above, the condition that the robot using the wheel having the passive rotating roller as the moving mechanism does not move by the passive rotating roller while the wheel is stationary is that the action vector of the passive rotating roller of at least one pair of wheels is It is not parallel. And there is no point where the passive rotation lines of all the wheels intersect in one place.

\section{Movement control of wheels with passive rotating rollers}

Consider the control of the drive wheels arranged under 2.3 "Conditions under which the robot using the wheel having the passive rotary roller as the moving mechanism does not move by the passive rotary roller while the wheel is stationary". The input information includes an orthogonal moving velocity vector $\overrightarrow{v_{m}}$ for the robot local coordinate fixed to the robot, a rotational moving angular velocity vector $\overrightarrow{\omega_{m}}$ for defining the rotational movement, a rotational axis position vector $\overrightarrow{p_{\omega}}$, and let the output be the rotational angular velocity $\omega_{i}$ (scalar) of each driving wheel.

\subsection{Definition of the position and driving direction of each wheel}

First, the position vector of the i-th driven wheel in the local coordinates fixed to the robot body and the unit vector in the direction of generating the velocity when the wheel is rotated in the positive direction are defined as follows. 


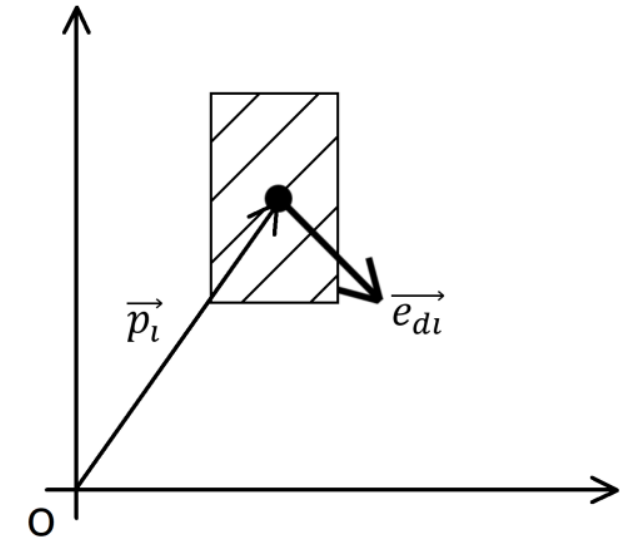

Fig. 4. Wheel placement parameters

$$
\begin{aligned}
& \overrightarrow{p_{\imath}}=\left[\begin{array}{l}
p_{x i} \\
p_{y i} \\
p_{z i}
\end{array}\right] \\
& \overrightarrow{e_{d l}}=\left[\begin{array}{l}
e_{x i} \\
e_{y i} \\
e_{z i}
\end{array}\right] \quad \text { where } \quad\left|\overrightarrow{e_{d l}}\right|=1
\end{aligned}
$$

Each drive has a position vector in the local coordinate of the robot and a unit direction vector in the direction in which the velocity is generated when the wheel is rotated. The moving speed of each wheel by the orthogonal moving speed vector

\subsection{The moving speed of each wheel by the orthogonal moving speed vector}

By the orthogonal movement speed vector $\overrightarrow{v_{m}}$, the velocity vector $\overrightarrow{v_{o}}$ generated on each wheel is added with the same velocity vector as the orthogonal movement velocity vector to each wheel regardless of the position of the wheel.

$$
\overrightarrow{v_{o}}=\overrightarrow{v_{m}}
$$

\subsection{Moving speed of each wheel by rotational angular velocity vector}

The speed generated on each wheel due to the rotational movement varies depending on the position of the driven wheel as viewed from the rotating shaft. The position of the driven wheel as viewed from the central axis of the rotational motion is expressed as follows.

$$
\overrightarrow{p_{l}}-\overrightarrow{p_{\omega}}
$$

The speed vector $\overrightarrow{v_{r}}$ generated on the wheel by the rotational movement angular velocity vector $\overrightarrow{\omega_{m}}$ is obtained by the cross product of the rotational movement angular velocity vector and the position vector of the wheel as viewed from the rotation axis, so it is as follows.

$$
\overrightarrow{v_{r}}=\overrightarrow{\omega_{m}} \times\left(\overrightarrow{p_{l}}-\overrightarrow{p_{\omega}}\right)
$$

\subsection{Synthesis of Cartesian and Rotational Travel Velocity}

Finally, the velocity vector applied to each wheel is the resultant vector of the velocity vector $\overrightarrow{v_{o}}$ derived from orthogonal motion and the velocity vector $\overrightarrow{v_{r}}$ derived from rotational movement, so the velocity vector $\mathrm{Vw}$ generated in each wheel is.

$$
\begin{aligned}
\overrightarrow{v_{w}} & =\overrightarrow{v_{o}}+\overrightarrow{v_{r}} \\
& =\overrightarrow{v_{o}}+\overrightarrow{\omega_{m}} \times\left(\overrightarrow{p_{l}}-\overrightarrow{p_{\omega}}\right)
\end{aligned}
$$

\subsection{Obtain the rotation speed $\omega_{i}$ of each wheel}

The speed vector hanging on the wheel is decomposed into the action direction $\overrightarrow{e_{p}}$ of the driven wheel and the drive action direction $\overrightarrow{e_{d l}}$. The speed component $v_{i}$ that can be generated by turning the wheel is considered to be the speed component of the velocity vector $\overrightarrow{v_{w}}$ applied to the wheel projected in the driving action direction $\overrightarrow{e_{d l}}$ (Fig. 5). That is, the speed at which each wheel should output is as follows.

$$
\begin{aligned}
v_{i} & =\overrightarrow{e_{d l}} \cdot \overrightarrow{v_{w}} \\
& =\overrightarrow{e_{d l}} \cdot\left(\overrightarrow{v_{o}}+\overrightarrow{v_{r}}\right) \\
& =\overrightarrow{e_{d l}} \cdot\left\{\overrightarrow{v_{o}}+\overrightarrow{\omega_{m}} \times\left(\overrightarrow{p_{l}}-\overrightarrow{p_{\omega}}\right)\right\}
\end{aligned}
$$

The target rotational speed can be obtained by converting the velocity component $v_{i}$ that can be generated by turning the wheel determined above into the wheel rotation speed $\omega_{i}$. In the case of the omni wheel, letting $r$ be the wheel radius. The rotation speed of the wheel is.

since $v_{i}=r \omega_{i}$

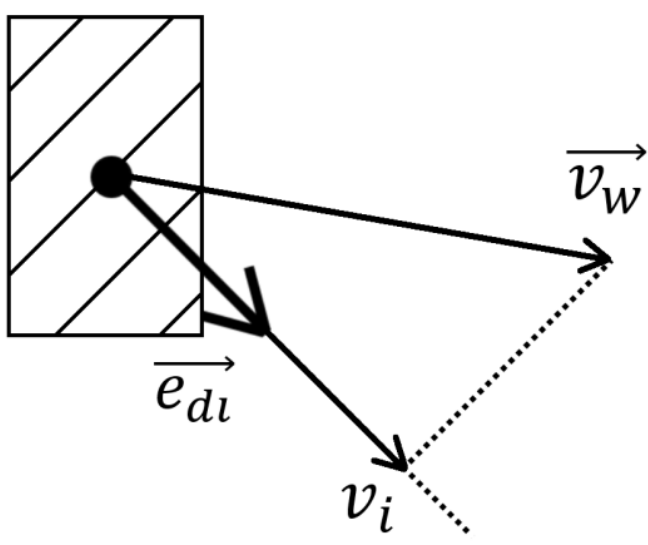

Fig. 5. Projection in driving direction 


$$
\omega_{i}=\frac{v_{i}}{r}
$$

In case of Mechanam Wheel.

$$
\begin{gathered}
\text { since } \quad v_{i}=\frac{1}{\sqrt{2}} r \omega_{i} \\
\omega_{i}=\sqrt{2} \frac{v_{i}}{r}
\end{gathered}
$$

Movement control of the wheel having the passive rotation roller can be performed by performing speed control using the obtained target rotation speed using a PID controller or the like.

\section{Drive compensation with IMU}

Using the IMU (inertial sensor), control the angle of the airframe. The correction of the body angle can be said to be an efficient correction method because it also leads to improvement of straightness as well as guarantee of angle.

\subsection{Angular estimation by angular velocity sensor}

The body angle of the robot is obtained by integrating the output of the angular velocity sensor. Since the offset is added to the output value of the gyro sensor, integration is performed by subtracting the offset.

\subsection{Adaptation to drive control equation}

Assuming that the target angle is $\theta_{t}$ and the current angle obtained by the angular velocity sensor is $\theta_{g}$, the angle correction can be applied to the drive control using the following expression. The rotational movement angular velocity vector $\overrightarrow{\omega_{m}}$ of the drive system is.

$$
\overrightarrow{\omega_{m}}=\left[\begin{array}{c}
0 \\
0 \\
\operatorname{pid}\left(\theta_{t}-\theta_{g}\right)
\end{array}\right]
$$

Here, pid(e) is the PID control block of the deviation input from the target, and the center of the rotational movement is defined to coincide with the center of the robot.

\section{Conclusion}

In this paper, we analyzed the characteristics of the robot using the wheel with passive rotating roller in the driving system, generalized the movement, and showed the method of correcting the aircraft angle using the IMU. From now on, the control equation In actual use, we evaluate the control of robot.

\section{References}

(1) Sato Minoru, Hamamatsu Yoshio, Hoshino Takahiro, "Feedback control by mobile encoder of mobile robot using Mechanum Wheel, Preliminary Workshop on Academic Lecture at Nihon University School of Science and Technology, L28 